\title{
Prótese bucomaxilofacial: a Odontologia além da boca
}

\author{
Maxillofacial prosthesis: the Dentistry beyond the mouth \\ Prótesis bucomaxilofacial: la Odontología allá de la boca \\ Gabriella Domingues DE CARVALHO ${ }^{1}$ \\ Liz Fernandes DE SOUZA ${ }^{\mathbf{1}}$ \\ Thaís Oliveira FERREIRA ${ }^{1}$ \\ Gabriela BENTO ${ }^{2}$ \\ Marcela Filié HADDAD ${ }^{3}$
}

\begin{abstract}
${ }^{1}$ Cirurgiã-Dentista pela Faculdade de Odontologia, Universidade Federal de Alfenas (UNIFAL-MG), 37.130-O00 Alfenas - MG, Brasil
${ }^{2}$ Cirurgiã-Dentista e Mestre pela Faculdade de Odontologia da Universidade Federal de Alfenas (UNIFAL-MG), 37.130-000 Alfenas - MG, Brasil

${ }^{3}$ Cirurgiã-Dentista, Mestre e Doutora em Prótese, Professora da disciplina de Prótese Parcial Removível do Departamento de Odontologia Restauradora, Faculdade de Odontologia, Universidade Federal de Alfenas (UNIFAL-MG), 37.130-000 Alfenas - MG, Brasil
\end{abstract}

\section{Resumo}

A prótese bucomaxilofacial é uma especialidade da Odontologia que visa restaurar indivíduos com perda de estrutura na região de boca e face. Seu ensino ainda é restrito na maioria dos cursos de Odontologia no Brasil, de modo que os cirurgiões dentistas se formam sem conhecer este ramo tão bonito da reabilitação e sem o domínio de conhecimentos teóricos e técnicos sobre o assunto. Deste modo, o objetivo do presente trabalho foi realizar uma revisão de literatura acerca da especialidade de prótese bucomaxilofacial para guiar os cirurgiõesdentistas a respeito de sua indicação, tipos, materiais utilizados e técnica de confecção.

Descritores: Prótese Maxilofacial; Prótese Dentária; Reabilitação.

\section{Abstract}

Maxillofacial prosthesis is a Dentistry specialty that aims to restore individuals with loss of structure in the mouth and face region. His teaching is still restricted in most of the Dentistry courses in Brazil, so that dental surgeons graduate without knowing this beautiful branch of rehabilitation and without mastery of theoretical and technical knowledge about the subject. Thus, the objective of the present study was to carry out a review of the literature on the specialty of maxillofacial prosthesis to guide dental surgeons regarding their indication, types, materials used and manufacture technique.

Descriptors: Maxillofacial Prosthesis; Dental Prosthesis; Rehabilitation.

\section{Resumen}

La prótesis bucomaxilofacial es una especialidad de la Odontología que busca restaurar individuos con pérdida de estructura en la región de boca y cara. Su enseñanza todavía está restringida en la mayoría de los cursos de Odontología en Brasil, de modo que los cirujanos dentistas se forman sin conocer esta rama tan hermosa de la rehabilitación y sin el dominio de conocimientos teóricos y técnicos sobre el tema. De este modo, el objetivo del presente trabajo fue realizar una revisión de literatura acerca de la especialidad de prótesis bucomaxilofacial para guiar a los cirujanos-dentistas acerca de su indicación, tipos, materiales utilizados y técnica de confección.

Descriptores: Prótesis Maxilofacial; Prótesis Dental; Rehabilitación.

\section{INTRODUÇÃO}

Pacientes com perdas de substâncias na região de cabeça e pescoço podem ser reabilitados proteticamente. As próteses bucomaxilofaciais visam restaurar partes da face, como: olho, nariz, orelha, região orbital, lábio ou da boca, como dentes, palato duro e mole, língua e osso. Entre as principais causas dessas perdas estão: traumatismos físicos e químicos, neoplasias, infecções, doenças autoimunes e defeitos congênitos ${ }^{1}$.

As deformidades na região de cabeça e pescoço podem resultar em fala incompreensível, dificuldade de mastigação, deglutição, sucção, respiração e audição do paciente, o que repercute social, emocional e psicologicamente, prejudicando a vida sexual, emprego, família e influenciando negativamente no bem estar da vítima. Mudanças profundas na subjetividade ocorrem e o rosto do paciente passa a ser menos atrativo aos olhares, sendo visto como anormalidade pelos outros e até mesmo pelo próprio indivíduo ${ }^{2}$. Muitas vezes a ausência das partes faciais é repulsiva, colocando o indivíduo em situações embaraçosas, tornando-o traumatizado e afastando-o do convívio social ${ }^{3}$.

A reposição das partes ausentes deve ser inserida no plano de tratamento e esse é um trabalho muito complexo, podendo ser feito mediante cirurgia plástica, peças protéticas ou ambas. Muitas vezes mutilações extensas não podem ser corrigidas cirurgicamente por falta de tecido doador suficiente, estado de saúde geral debilitado ou porque o resultado estético e funcional será comprometido. Nesses casos os defeitos deverão ser substituídos artificialmente por próteses bucomaxilofaciais ${ }^{4}$. Estes dispositivos protéticos trazem como vantagens a possibilidade de observação da cicatrização da ferida, avaliação da recorrência da doença, simplicidade da técnica, custo reduzido e, em alguns casos, superioridade estética ${ }^{5}$.

A reparação da perda facial, através da prótese bucomaxilofacial, além de restaurar a aparência, função e proteger os tecidos expostos, também possui como finalidade reduzir o sentimento de ansiedade, possibilitando o soerguimento da autoimagem e autoestima do paciente e a promoção da sua reintegração ao convívio social. Contudo, se essas alterações não forem trabalhadas psicologicamente, a reabilitação protética não alcançará por completo seus objetivos ${ }^{6}$. Por isso o tratamento terapêutico do paciente em questão deve envolver equipe multidisciplinar e o conhecimento da patogênese da lesão para planejamento correto e sucesso na reabilitação ${ }^{7}$.

O propósito deste trabalho foi realizar uma revisão de literatura acerca da especialidade de prótese bucomaxilofacial para guiar os cirurgiões-dentistas a respeito de sua indicação, tipos, 
materiais utilizados e técnica de confecção.

\section{REVISÃO DA LITERATURA}

\section{- Perdas bucomaxilofaciais - etiologia}

Entre as grandes causas das perdas bucomaxilofaciais estão: traumatismos físicos (ex.: acidentes por armas de fogo, armas brancas, automobilísticos, violência em geral), químicos (ex.: substâncias corrosivas), neoplasias (ex.: Carcinoma de Células Escamosas, Carcinoma Espinocelular, Adenocarcinoma das Glândulas, entre outros), infecções fúngicas (ex.: Paracoccidioidomicose, Aspergilose, etc.), bacterianas (Sífilis, Lepra, Tuberculose e Actinomicoses) ou parasitárias (ex.: Leishmaniose, Miíase, Mononucleose, etc.), doenças autoimunes (ex.: Lúpus Eritematoso) e defeitos congênitos (ex. Fissura Nasopalatina, Fissura Nasolabial, etc. $)^{2,5,6}$.

As neoplasias representam a etiologia mais incidente das deformidades bucomaxilofaciais e, em sua grande maioria, são diagnosticadas em fase avançada, implicando em tratamento extremamente mutilador e com baixa sobrevida ${ }^{2,6}$.

\section{- Reabilitação de defeitos bucomaxilofaciais}

A reabilitação dos defeitos bucomaxilofaciais pode se dar por meio de cirurgias reconstrutoras ou dispositivos protéticos. A escolha do método de reabilitação vai depender da etiologia, gravidade e local da perda, tamanho do defeito, idade, estado sistêmico e preferência do paciente ${ }^{8}$.

A reconstrução cirúrgica é desejável, sendo a primeira opção para a reabilitação de pacientes com mutilações na região bucomaxilofacial, porém, este método possui alguns limitantes, tais como: idade, estado de saúde geral do paciente, tecido residual insuficiente, necessidade de acompanhamento da recorrência de tumores, comprometimento vascular e inadequação do local de doação. Assim, a reabilitação protética se torna uma opção viável ${ }^{9}$.

As próteses bucomaxilofaciais podem ser de diferentes tipos, de acordo com a área que elas reabilitam e, assim, são divididas em próteses oculares, nasais, oculopalpebrais, auriculares, maxilofaciais, obturadoras palatinas e faringeanas, podendo ser utilizadas de maneira individual ou conjugada ${ }^{9}$.

- Materiais e técnicas de confecção de próteses bucomaxilofaciais

A especialidade de Prótese Bucomaxilofacial é uma das mais complexas da odontologia, pois deve restaurar não apenas a função e anatomia, mas também ser o mais natural possível. Por conta disso a busca por materiais que muito se assemelham ao tecido e possuam os requisitos necessários torna-se cada vez mais importante. Além disso, o resultado é subjetivo, pois leva em consideração os padrões estéticos de cada profissional, a escolha adequada da técnica e dos materiais a serem utilizados, fornecendo um bom resultado e reabilitando o paciente funcional, estética e emocionalmente. A Prótese Bucomaxilofacial exige profissionais com ampla visão clínica e que atuem em equipes multidisciplinares, pois o tratamento deve ser conduzido de forma sincronizada ${ }^{10}$.

\section{$>$ Próteses obturadoras}

A maxilectomia sempre acarreta deficiência de funcionalidade na cavidade bucal, seja ela parcial ou total. As comunicações geradas devem ser fechadas e para este fim utiliza-se obturador palatino, que é um dispositivo protético que substitui a área ausente, obliterando a comunicação existente. Este dispositivo pode estar associado à armação de prótese parcial removível ou a base de uma prótese total ou parcial removível. Com a confecção e instalação de tal dispositivo as funções orais são reestabelecidas e a face ganha suporte novamente, permitindo que seu contorno volte ao normal, tornando possível a recuperação física e psicológica do paciente ${ }^{11}$.

$$
\text { Grandes perdas maxilares agem }
$$

negativamente na biomecânica das próteses obturadoras. As forças de alavanca e linhas de fulcro aumentam a força sobre os tecidos de suporte durante a mastigação. $\mathrm{O}$ peso intrínseco da prótese obturadora sobre os tecidos de suporte representa um fator agravante, havendo necessidade de adequada avaliação dos tecidos remanescentes para o bom desempenho da prótese e preservação das estruturas anatômicas de retenção e suporte. A retenção dessa prótese é proporcionada pelas estruturas presentes na maxila, que se limita aos dentes remanescentes, já que as bordas do defeito cirúrgico não são levadas em consideração. Com isso, o número, localização e as condições periodontais são fatores cruciais na avaliação da quantidade de tensão que poderão absorver. Os dentes posicionados na borda do defeito cirúrgico estão sujeitos a forças rotacionais que excedem o limiar de absorção fisiológica e que podem acarretar a perda prematura de dentes retentores ${ }^{12}$.

As maxilectomias podem ser uni ou bilaterais. No caso das unilaterais a preservação do incisivo central contra lateral, quando possível, otimiza o resultado estético e funcional, uma vez que esse servirá de ancoragem para o aparelho. No caso de maxilectomia bilateral, a impossibilidade de preservação de elementos que poderiam auxiliar na sustentação e reconstrução da função e estética constitui um grande desafio na reabilitação do paciente em questão, desafio esse que se torna maior também em pacientes edentados ${ }^{13}$.

Em situações em que todos ou alguns dentes do paciente são mantidos, estes devem ser utilizados para promover a retenção do aparelho protético, que deve ter uma infraestrutura metálica assim como as próteses parciais removíveis, porém, apresentando o bulbo obturador. Para isso nichos devem ser 
preparados nos dentes pilares a fim de acomodar os apoios dos grampos e o delineamento e planejamento da armação metálica devem ser realizados da mesma maneira que as próteses parciais removíveis convencionais ${ }^{14}$.

No que se refere à confecção da prótese obturadora, são necessárias duas moldagens distintas: uma de estudo e outra de trabalho. O modelo de estudo objetiva a confecção de moldeira individual, delineamento e avaliação da necessidade de alteração de contorno (quando se trata de arcadas parcialmente desdentadas); já a moldagem de trabalho possui como finalidade a obtenção de um modelo funcional, sobre o qual será confeccionada a base de prova (no caso de arcadas completamente desdentadas) ou a armação metálica (em caso de arcadas parcialmente desdentadas) ${ }^{15}$. Para estas moldagens podem ser utilizados alginato ou siliconas, sendo que as siliconas apresentam melhores características de reprodutibilidade ${ }^{16}$.

Sobre o modelo funcional da prótese total obturadora é confeccionada a base de prova com o plano de cera e, em seguida, as linhas de referências para a seleção dos dentes artificiais são marcadas. Nos casos de próteses parciais removíveis obturadoras, a partir do modelo de trabalho é confeccionada a armação metálica, que é provada na boca do paciente, recebe os ajustes necessários e, sobre ela, também é confeccionada base de prova e plano de cera na região desdentada. Em seguida, os registros intermaxilares são tomados e os modelos montados no articulador. Na etapa seguinte realiza-se a montagem dos dentes artificiais e prova estética e funcional, sempre observando fala e respiração ${ }^{15}$.

Para confecção da base das próteses obturadoras os seguintes materiais podem ser utilizados: resinas acrílicas auto, termo ou fotopolimerizáveis, resina soft (para reembasamento), Aquaplast/thermoplast e também placa de acetato (para obturador confeccionado na fase transcirúrgica $)^{11}$. Atualmente o material mais utilizado no Brasil para confecção das próteses obturadoras definitivas é a resina de metilmetacrilato termopolimerizável devido ao seu fácil manuseio, baixo custo, grande oferta, durabilidade e por ser higiênica, porém, é limitada por sua rigidez ${ }^{17}$.

Para cada defeito maxilar há uma possibilidade de confecção de um obturador palatino, o que depende da extensão, presença ou ausência de estrutura de suporte e das condições gerais de saúde. Às vezes é necessária a confecção de retenções adicionais para a estabilidade da prótese e para que a mesma fique em posição. Além disso, a prótese deve ser leve para que a força da gravidade não a desloque $^{11}$.

Em casos em que o defeito cirúrgico é expulsivo, característica desfavorável, dispositivos devem ser utilizados para retenção e estabilidade da prótese. São eles: implantes associados a magnetos/encaixes ou molas presas a banda ortodôntica nos molares ou na prótese inferior ${ }^{11}$.

\section{$>$ Próteses maxilofaciais}

Para a confecção da prótese bucomaxilofacial é necessário um modelo, que é a reprodução do defeito, e serve de guia para a reabilitação. Sua obtenção é extremamente necessária para a escultura da prótese e para que a mesma se adapte corretamente aos tecidos remanescentes. Para execução da moldagem é preciso um material que possa ser aplicado sobre a região a ser modelada, de presa rápida, que se adapte e que possa ser removido sem distorções. Outras propriedades importantes são: baixa viscosidade, estabilidade dimensional, elasticidade suficiente, envolver o mínimo de aplicação de massa na replicação do tecido a ser moldado para que os tecidos moles não sofram deformação pelo seu peso ${ }^{18}$.

No que diz respeito à moldagem para confecção da prótese facial, um dos requisitos iniciais para sua realização consiste na informação ao paciente de que haverá um pouco de dificuldade para respirar, ausência de luz pela presença do material sobre seus olhos e, ainda, o local de trabalho deve estar calmo, arejado e com ausência de ruídos para maior tranquilidade do paciente, que deve ser posicionado em decúbito dorsal com o tronco e a cabeça soerguidos de $30^{\circ}$ em relação ao plano horizontal para minimizar os efeitos gravitacionais nos tecidos adjacentes à área de interesse ${ }^{18}$.

A técnica convencional para confecção do modelo facial utiliza como material de moldagem o hidrocolóide irreversível (alginato) apoiado por uma base de gesso. Este material possui como características o baixo custo, facilidade na mistura e manipulação e a formação de um conjunto flexível após a geleificação. Porém, seu tempo de trabalho é limitado. Nesta técnica a pele do paciente deve estar limpa, os cabelos protegidos com gorro descartável e as regiões pilosas e áreas cruentas isoladas com vaselina para que a remoção do molde seja facilitada. Deve-se manipular o alginato e levá-lo em pequenas porções na área a ser moldada com uma espátula. Em seguida, deve-se fazer retenção entre o alginato e o gesso com grampos metálicos em $\mathrm{U}$ nos arredores do molde ou gaze ou algodão sobre o alginato antes de sua geleificação. Em seguida insere-se uma camada de gesso tipo II sobre o alginato para fornecer uma base rígida para a remoção do molde. A remoção do molde é feita com movimentos leves e cuidadosos de vibração de cima para baixo e de trás para frente pedindo ao paciente que movimente os músculos mímicos. Avalia-se, então o molde. Após a moldagem, o molde deve ser preenchido com gesso para obtenção do modelo ${ }^{18}$.

Existem várias outras técnicas e materiais para moldagem da máscara facial, tais como: 
silicones, associação do polivinilsiloxino e do hidrocolóide irreversível com uma moldeira personalizada, CAD/CAM, sensor tridimensional a laser associado a uma máquina de prototipagem rápida, sistema de imagens fotográficas em três dimensões, porém, estas técnicas possuem custo mais elevado ou necessitam de treinamento do profissional, o que as tornam mais onerosas ${ }^{18}$.

Reconstruir o defeito facial é uma tarefa complexa, pois exige como requisitos conhecimento anatômico, dons artísticos e, ainda, conhecimento dos materiais utilizados para a confecção da prótese ${ }^{19}$. A escultura da região do defeito objetiva copiar fielmente todas as linhas de expressão e irregularidades da pele. A abertura palpebral é confeccionada tomando como referência o olho saudável do paciente. Os limites da prótese devem ser camuflados em rugas e saliências naturais do paciente para obtenção de maior naturalidade no que se refere à aparência. As próteses faciais podem ser esculpidas em cera, argila ou massa de modelar. A escolha do material a ser utilizado, deve ser baseada na capacidade de cada operador em dar forma ao material $^{20}$.

Feitas todas as adaptações necessárias, a escultura da prótese citada acima é incluída em mufla $^{15}$. Após a inclusão da prótese facial em mufla e cristalização do gesso, constitui passo subsequente a remoção da cera e o preenchimento do molde deixado por ela com material estético. Os materiais de escolha para confecção deste tipo de prótese são as resinas acrílicas ativadas termicamente e silicones polimerizados pelo calor ou à temperatura ambiente. Contudo, a resina acrílica não possui flexibilidade, o que é essencial para que a prótese seja confortável ao paciente, fazendo do silicone o material mais próximo do ideal, uma vez que este apresenta semelhança com a textura da pele, pequena espessura das margens da prótese, facilidade de pigmentação e maior conforto. As próteses faciais confeccionadas em silicone apresentam limitações como: descoloração, dificuldade de reparo, tempo de vida útil reduzido e degradação das propriedades físicas ${ }^{21}$.

A pigmentação da prótese facial pode ser realizada utilizando pigmentos orgânicos e inorgânicos, sendo os últimos mais estáveis em relação à cor, especialmente quando associados a agentes opacificadores. Um dos exemplos de opacificador é o sulfato de bário ${ }^{21}$.

As próteses faciais possuem como forma de retenção adesivos, rebaixados anatômicos, óculos ou ímãs, porém, o uso de implantes para retenção nas regiões craniofaciais podem eliminar ou minimizar a necessidade de adesivos, permitindo orientação e assentamento adequado quando o planejamento é feito de forma cuidadosa em termos de número, posição e orientação dos implantes, além da ligação correta da prótese à estrutura de retenção do implante. Com isto a estética e durabilidade da prótese são melhoradas, já que as margens são finas, de fácil manutenção e não susceptíveis aos prejuízos dos solventes dos adesivos. Entretanto, pacientes oncológicos muitas vezes podem passar por tratamento radioterápico e por isso o osso para colocação do implante pode ser comprometido ou perdido, já que o potencial osteogênico do mesmo e microvascularização são diminuídos ${ }^{22}$.

Constituem tipos de retenção: anatômica (uso de estrutura anatômica existente), mecânica (armação de óculos, peças acrílicas, clips, magnetos e botões), adesiva (adesivos apropriados aplicados à prótese $\mathrm{e}$ fixada à pele ou, ainda, fitas adesivas dupla face, estas em casos provisórios de retenção) e cirúrgica (implantes unitários ou agrupados) ${ }^{15}$.

As próteses oculares e bucais geralmente conseguem uma boa retenção anatômica. Já as faciais, auriculares, nasais e óculo-palpebrais necessitam de retenção adicional por meio de adesivos ou implantes. Apesar de a retenção adesiva apresentar muitos incômodos, como ineficácia na retenção, inflamação da pele e corrosão da prótese, a maioria dos pacientes usa esse tipo de retenção ${ }^{5}$.

- Higienização das próteses bucomaxilofaciais

Um fator importante no que diz respeito à escolha do material constituinte do aparelho protético é a possibilidade de higienização, já que o contato desse com a pele ocorrerá de forma constante. Para que a longevidade da prótese seja suficiente, os cuidados referentes à desinfecção, colocação e remoção da prótese bucomaxilofacial, bem como a proservação do tratamento são fundamentais ${ }^{21}$.

A higiene oral e da prótese obturadora são essenciais para manter a saúde dos tecidos, prevenindo o desenvolvimento de patologias, como cárie dentária, periodontopatias e processos inflamatórios da mucosa, além de aumentar a longevidade da prótese. Essa higienização é dividida em dois métodos: mecânicos e químicos. O mecânico consiste na escovação com água e sabão ou dentifrício e deve ser feito com escova apropriada e pasta pouco abrasiva, a fim de evitar desgastes na resina acrílica. Quando bem realizada, é efetiva na remoção de manchas artificiais. Já o químico lança mão de peróxidos alcalinos, que estão disponíveis em forma de pó ou tablete e se transformam em soluções quando são dissolvidos em água ${ }^{23}$.

Próteses maxilofaciais confeccionadas com silicone podem apresentar colonização por biofilme de múltiplas espécies, por isso, nos casos de dificuldade de higienização, a utilização de um antifúngico é indicada ${ }^{21}$.

Quanto às técnicas para realização da limpeza das próteses faciais, a escovação não é aconselhável, pois sua frequente execução dissolve e remove pigmentos da superfície externa. Enxaguar em água corrente não é um procedimento eficaz 
contra formação de manchas. O melhor método para limpeza consiste na saturação química, sendo recomendado aos pacientes que os mesmos não limpem a prótese com qualquer solvente que pode causar dissolução de pigmentos e degradação do silicone $^{21}$.

\section{- Durabilidade das próteses bucomaxilofaciais}

Há necessidade de troca das próteses ao longo da vida e os motivos variam entre desgaste da prótese, aumento da cavidade ou ambos. Alguns pacientes são submetidos a reconstruções muito novos e, com o crescimento, há necessidade de substituição das próteses para que elas fiquem proporcionais em um curto período de tempo. A vida útil de uma prótese confeccionada em resina acrílica é de mais ou menos cinco anos, após isso ela começa apresentar deterioração e irritação dos tecidos. O silicone apresenta grande alteração cromática, o que leva a necessidade de substituições mais precoces. As próteses bucais precisam ser substituídas a partir do momento em que não apresentam mais uma boa retenção e/ou que começam a deixar os usuários insatisfeitos com alguma alteração ${ }^{9}$. A cooperação do paciente é de extrema importância para a preservação de sua saúde, seus tecidos e aumento da vida útil de sua prótese. Consultas trimestrais e anuais devem ser realizadas para controle da manutenção ${ }^{24}$.

\section{DISCUSSÃO}

A prótese bucomaxilofacial representa um grande desafio dentro das especialidades odontológicas, restaurando partes ou regiões da face e levando em consideração aspectos anatômicos e funcionais, de modo a reproduzi-los com a maior naturalidade possível. A atuação dessa especialidade vem reafirmar o conceito de saúde, cujo objetivo final deve ser o bem-estar social, físico e mental do paciente, não apenas a inexistência de enfermidade ${ }^{10}$.

Resultados de excelência dependem diretamente do discernimento do cirurgião dentista no que diz respeito à escolha de materiais adequados e até mesmo adoção de técnicas laboratoriais corretas. Além disso, os aspectos emocionais do paciente mutilado devem ser levados em consideração, o que culmina na maior colaboração e aceitação do paciente, tão desejados para uma reabilitação de alta complexidade ${ }^{10}$.

No que diz respeito à confecção da prótese obturadora, esta segue os princípios de uma prótese parcial ou total removível, cuja técnica é simples, rápida, eficaz, passível de adaptações e de fácil instalação. A confecção deste tipo de prótese destinase a pacientes que necessitam de correção de defeitos estéticos e funcionais e que não podem optar por cirurgias reconstrutivas ${ }^{11}$.

Para execução dessa prótese, pode-se utilizar resina acrílica auto, termo ou fotopolimerizável, resina soft, Aquaplast/thermoplast e placa de acetado $^{11}$. O material de escolha é a resina acrílica termopolimerizável por apresentar as seguintes vantagens: fácil manuseio, baixo custo, grande oferta, durabilidade e facilidade de higienização. Como limitação pode ser apontada sua rigidez, que pode injuriar tecidos se não estiver bem ajustada e polida ou ainda se os tecidos de suporte ainda estiverem em cicatrização $^{17}$.

As instruções ao paciente quanto à higienização, alimentação adequada no início do uso da prótese obturadora e como utilizá-la são de extrema importância para o sucesso do tratamento e para que o paciente não desista do uso das próteses ${ }^{21}$.

Para a confecção das próteses maxilofaciais definitivas os materiais mais utilizados são resina acrílica e silicone. A resina acrílica apresenta alta rigidez, não conseguindo mimetizar a textura da pele, ficando sua indicação restrita a trabalhadores rurais, que sofrem exposição solar diária e prolongada, fator este que provoca deterioração do silicone em curto período de tempo; para casos de próteses muito extensas, retidas por múltiplos implantes, uma vez que a adesão dos sistemas de retenção dos implantes ao silicone é precária; e em casos em que o leito protético não apresente muita movimentação. Para as demais situações o silicone é o material de escolha para a confecção destas próteses devido à facilidade de pigmentação e de manipulação, biocompatibilidade e, sobretudo, por possuir textura e elasticidade semelhantes a da pele, sendo indicados para leitos de maior movimentação, garantindo maior biomimetismo ${ }^{5,8,21}$.

Quanto à retenção destas próteses faciais, podem ser citados os meios anatômicos, mecânicos e adesivos. Todas as próteses dessa categoria se valem, mesmo que parcialmente, da retenção anatômica, que consiste explorar o defeito existente para ajudar na retenção e estabilização do dispositivo protético. Os meios mecânicos incluem a associação de dispositivos externos, tais como armações de óculos, molas, clipes, ímãs e até mesmo implantes à prótese. A retenção adesiva se dá através da aplicação de adesivos próprios para a pele na superfície da prótese $^{2,5,9,11,12}$.

Os implantes osseointegráveis representam uma forma de retenção para as próteses bucomaxilofaciais, pois permite que as mesmas fiquem mais leves, com bordas mais finas, sem o efeito deletério do solvente aplicado para remoção do adesivo, o que culmina em melhoria funcional, social e psicológica para o paciente. Além da boa retenção protética, o acesso clínico para verificar a recorrência de tumores malignos e aceitabilidade cosmética representam aspectos favoráveis ${ }^{24}$.

Porém, a radioterapia, apesar de constituir arma eficaz para o controle de tumores, pode levar a perda de implantes, deiscência de tecidos moles e até mesmo osteorradionecrose. A instalação de 
implantes, por sua vez, muitas vezes precisa ser realizada em áreas que passaram por processo de radiação, o que torna esse tipo de retenção inviável em muitos casos. Assim, o indivíduo irradiado deve ser tratado com maior cautela, levando em consideração os efeitos biológicos do tratamento radioterápico, idade, dose, local, tempo de radiação, possibilidade de falência dos implantes e as possíveis complicações agudas tardias ${ }^{24}$.

Em caso de próteses conjugadas, a retenção entre a prótese obturadora e a maxilofacial comumente se dá através da conexão magnética, constituída por ímã na região do bulbo e seu análogo na região da prótese facial. Esta forma de retenção apresenta-se como uma opção viável para o tratamento reabilitador, pois se mostra capaz de fornecer retenção adequada à prótese, principalmente com desenvolvimento de ímãs com menor dimensão e maior capacidade magnética. No que diz respeito à desvantagem, o ímã pode sofrer, quando em contato com os fluidos teciduais, corrosão, que culmina na diminuição ou inexistência deste tipo de retenção ${ }^{9,15}$.

Outra forma de retenção para próteses bucomaxilofaciais são os adesivos, que são aplicados sobre a superfície da prótese que, em seguida, é fixada à pele. Esse tipo de retenção tem grande importância pelo seu baixo custo e facilidade operacional, principalmente quando outros tipos de retenções não são indicadas. Porém, existem desvantagens significativas para o uso de adesivos, sendo elas: as margens da prótese facial podem ser danificadas pela sua aplicação repetidas vezes e o paciente poderá desenvolver reação alérgica. Além disso, eles podem perder sua função em ambientes úmidos e possuem dificuldades relacionadas com retenção, deterioração da estabilidade, descoloração da prótese, inconveniência de uso ou aplicação, falta de higiene, desconforto e aceitabilidade ${ }^{25}$.

Apesar da complexidade da execução dos passos clínicos e laboratoriais, além do prognóstico nem sempre favorável, as próteses bucomaxilofaciais conjugadas constituem excelente opção para casos complexos de mutilação e costumam satisfazer as necessidades do paciente de forma aceitável, além de representar uma possibilidade de reinclusão social do indivíduo $^{1,2,5,21}$.

\section{CONCLUSÃO}

A prótese bucomaxilofacial é uma opção para indivíduos que possuem elevado grau de mutilação de modo a devolver, mesmo que parcialmente, a estética, função, convívio social e autopercepção. Além disso, ela permite o constante inspecionamento da região acometida pelo tumor de modo a detectar possíveis recidivas.

Espera-se que, a partir da leitura do presente relato, os Cirurgiões Dentistas tomem conhecimento das etapas clínicas e laboratoriais necessárias para a confecção de próteses bucomaxilofaciais conjugadas e conheçam a importância deste tipo de reabilitação protética quanto à estética, função, melhora no estado emocional e autopercepção do indivíduo reabilitado.

Espera-se, também, que os médicos tomem conhecimento da existência deste tipo de reabilitação e façam o planejamento da etapa cirúrgica em conjunto com o planejamento da reabilitação protética.

\section{REFERÊNCIAS}

1. Alfenas ER, Lanza CRM, Barreiros ID, Novaes Junior JB, Mendonça LL. Reabilitação protética do paciente com perda de substância na região de cabeça e pescoço. Arq Odontol. 2011;47(2):28-31.

2. Dos Santos DM, de Caxias FP, Bitencourt SB, Turcio KH, Pesqueira AA, Goiato MC.Oral rehabilitation of patients after maxillectomy. A systematic review. Br J Oral Maxillofac Surg. 2018;56(4):256-66.

3. Gamarra RS, Oliveira JAP, Dib LL. A estética em reabilitação bucomaxilofacial. Rev APCD. 2015; 3(1):42-52.

4. de Oliveira FM, Salazar-Gamarra R, Öhman D, Nannmark U, Pecorari V, Dib LL. Quality of life assessment of patients utilizing orbital implant-supported prostheses. Clin Implant Dent Relat Res. 2018;20(4):438-43.

5. Goiato MC, Tabata LF, Archangelo CM, Júnior MM. Uso de implantes ossointegrados associados a sistemas de retenção na reabilitação com próteses bucomaxilofaciais: Revisão de Literatura. Pesq Bras Odontoped Clín Integr. 2007;7(3):231-36.

6. Goiato MC, de Carvalho Dekon SF, de Faria Almeida DA, Sánchez DM, dos Santos DM, Pellizzer EP. Patients' satisfaction after surgical facial reconstruction or after rehabilitation with maxillofacial prosthesis. J Craniofac Surg. 2011;22(2):766-9.

7. Kusterer LEFL, Paraguassú GM, Silva VSM, Sarmento VA. Reabilitação com obturador maxilar após cirurgia oncológica: relato de casos. Rev Cir Traumatol Buco-Maxilo-Fac. 2012;12(4):9-16.

8. Nemli SK, Aydin C, Yilmaz H, Bal BT, Arici YK. Quality of life of patients with implant-retained maxillofacial prostheses: a prospective and retrospective study, J Prosthet Dent. 2013;109(1):44-52.

9. Goiato MC, Pesqueira A.A, Ramos da Silva C, Gennari Filho H, Micheline dos Santos D. Patient satisfaction with maxillofacial prosthesis. Literature review. J Plast Reconstr Aesthet Surg. 2009;62(2):175-80.

10.Simões FG, Reis RC, Dias RB. A especialidade de prótese bucomaxilofacial e sua atuação na Odontologia. Rev Sul Bras Odontol. 2009;6(3):327-31. 
11. Aguiar L, Mozzini AR, Lersch E, De Conto F. Obturador palatino: confecção de uma prótese não convencional - relato de caso. Rev Fac Odontol Passo Fundo. 2013;18(1):125-29.

12. Miyashita ER, Mattos BSC, Marafon PG. Sistemas de retenção em prótese obturadora cirúrgica para pacientes maxilectomizados. Pesq Bras Odontoped Clín Integr. 2011;11(2):263-68.

13.Esteves AJ, Costa FCM, Haddad MF. Prosthetic rehabilitation of oncological patient: case report. Arch Health Invest. 2016;5(6): 291-97.

14. Silva DP, Almeida FC, Vaccarezza GF, Brandão TB, Cazal C, Caroli A et al. Reabilitação protética de pacientes maxilectomizados. Uma contribuição da odontologia e um convite à reflexão. Pesq Bras Odontoped Clín Integr. 2004;4(2):125-30.

15.Rezende JRV. Fundamentos da prótese bucomaxilo-facial. São Paulo: Savier, 1997; 1:1-10.

16. Tucci R, Antonio LFM, de Carvalhosa AA, Catro PHS, Nunes FD, Pinto Junior DS1. Central mucoepidermoid carcinoma: report of a case with 11 years' evolution and peculiar macroscopical and clinical characteristics. Med Oral Patol Oral Cir Bucal. 2009;14(6):283-86.

17.Sperb LCM, Neves ACC, Rode SM. Considerações sobre prótese ocular: Sua importância na odontologia atual. RGO. 2001; 49(4):202-24.

18. Aquino LMM, Oliveira M, Martins APVB, Barbosa CMR. Técnicas de moldagem da máscara facial. Rev Odontol UNESP. 2012;41(6):438-41.

19. Neves ACC, Murgo DA, Campoy CD, Coas VR. Prótese facial combinada. RGO. 2005;53(1):1-4.

20. Sousa AVB, Arriaga MH, Llorca FA, Henar TEl. Prótesis oculo-palpebral. A propósito de un caso clínico. RCOE. 2003;8(5):553-61.

21.Filié Haddad M, Coelho Goiato M, Micheline Dos Santos D, Moreno A, Filipe D'almeida N, Alves Pesqueira A. Color stability of maxillofacial silicone with nanoparticle pigment and opacifier submitted to disinfection and artificial aging. $\mathbf{J}$ Biomed Opt. 2011;16(9):095004.

22.Abu-Serriah M, McGowan D, Moos K, Bagg J. Extra-oral craniofacial endosseous implants and radiotherapy. Int $\mathrm{J}$ Oral maxillofac Surg. 2003;32(6):585-92.

23. Silva RJ, Seixas ZA. Materiais e métodos de higienização para próteses removíveis. Int J Dent. 2008;7(2):125-32.

24. Antunes AA, Carvalho RWF, Lucas Neto A, Loretto NRM, Silva EDO. Utilização de implantes osseointegrados para retenção de próteses bucomaxilo-faciais: revisão de literatura. Rev Cir Traumatol Buco-Maxilo-Fac. 2008;8(2):9-14.

25. Haddad MF, Goiato MC, Santos DM, Crepaldi Nde M, Pesqueira AA, Bannwart LC. Bond strength between acrylic resin and maxillofacial silicone. J Appl Oral Sci. 2012;20(6):649-54.
CONFLITO DE INTERESSES

Os autores declaram não haver conflitos de interesse.

AUTOR PARA CORRESPONDÊNCIA

Marcela Filié Haddad

marcela.haddad@unifal-mg.edu.br

Submetido em 02/10/2018

Aceito em 17/01/2018 